Passenger

\section{Top 20}

Rank

World

1 United States

2 Soviet Union

3 Japan

4 United Kingdom

5 Canada

6 France

7 Australia

8 Germany, West

9 Spain

10 Italy

11 Netherlands

12 Brazil

13 Switzerland

14 Mexico

15 India

16 Singapore

17 South Africa

18 Thailand

19 Greece

20 Korea, South

Rest of the world ${ }^{d}$

\section{Rank}

in 1976

21 Israel

22 New Zealand

23 Argentina

24 Sweden ${ }^{k}$

26 Pakistan

27 Norway

28 Saudi Arabia

29 Indonesia

30 Iran

31 Philippines

32 Colombia

33 Taiwan

34 Portugal

35 Hongkong

6 Denmark

37 Venezuela

38 Jugoslavia

39 Turkey

40 Iceland

41 Algeria

42 Malaysia

43 Lebanon

44 Egypt

45 Ireland

46 Germany, East

47 Poland

48 Finland

49 Jamaica

50 Peru

51 Czechoslovakia

52 Morocco

53 Chile

54 Kuwait

5 Trinidad \& Tobago 104

56 Tunisia 968

57 Kenya $^{\text {m }} \quad 879$

58 Iraq 863

59 Austria

60 Jordan
Passenger-km (mn)

1970

1976

Growth \%pa

$21000 \quad 460000 \quad 763000$

$\begin{array}{rrr}62542 & 210327 & 288027 \\ 12111 & 78226 & 130529\end{array}$

$\begin{array}{lll}1051 & 14954 & 32334\end{array}$

$\begin{array}{lll}6372 & 17432 & 30948\end{array}$

$4267 \quad 15397 \quad 26031$

$5229 \quad 13587 \quad 25192$

$\begin{array}{lll}3008 & 9268 & 19384\end{array}$

$\begin{array}{lll}1284 & 8255 & 14982\end{array}$

11130

$\begin{array}{ll}5874 & 11130 \\ 8395 & 10780\end{array}$

$5769 \quad 10613$

$4385 \quad 10366$

$4420 \quad 8493$

$2939 \quad 7833$

$\begin{array}{lll}1115 & 3555 & 7196\end{array}$

$190^{\text {h.j }} \quad 664^{\mathrm{J}} \quad 6362$

$513 \quad 2872$

783

2126
445
14.3

$\begin{array}{ll}14.3 & 8.8 \\ 12.9 & 5.4\end{array}$

20.5

30.4

$10.6 \quad 10.0$

$13.7 \quad 9.1$

$10.0 \quad 10.8$

$11.9 \quad 13.1$

$20.5 \quad 10.4$

$22.3 \quad 11.2$

$20.0 \quad 4.3$

$8.0 \quad 10.7$

$5.1-15.4$

$\begin{array}{rr}14.5 & 11.5 \\ 8.4 & 17.7\end{array}$

$12.3 \quad 12.5$

$36.71 \quad 45.7$

$18.8 \quad 13.2$

$28.6 \quad 34.6$

$\begin{array}{ll}22.1 & 13.8 \\ 34.9 & 47.2\end{array}$

\section{Pass-km Rank}

(mn) 1976

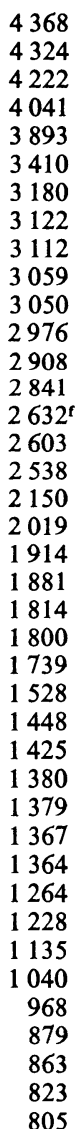

in 1976

61 Rumania

62 Syria

63 Libya

64 Zaire

65 Cuba

66 Ethiopia

67 Hungary

68 Bulgaria

69 Dominican Rep

70 Nigeria

71 Bolivia

72 Panama

73 Bangladesh

74 Bahamas

75 Zambia

76 Sudan

77 Malta

78 Costa Rica

$79=$ Bahrain $^{8}$

$79=$ Oman $^{8}$

79= Qatar

$79=$ Utd Arab Emirates

83 Ecuador

84 Mozambique

85 Sri Lanka

86 Cyprus

87 Madagascar

88 Afghanistan

89 Honduras

90 Barbados

91 Yemen, North

92 Cameroon

93 Papua New Guinea

94 Uganda $^{m}$

$5=$ El Salvador

95 $=$ Tanzania $^{\mathrm{m}}$

97 Ghana

98 Gabon $^{1}$

99 Burma

100 Luxembourg
Pass-km

(mn) 1976

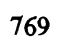

712

700

690

663

523

510

500

487

470

444

437

426

402

356

345

340

326

320

320

320

320

318

312

305

304

276

258

257

251

237

225

220

197

195

195
194

$190^{*}$

168
Cargot

Top 20

Rank

Tonne-km, net (mn) Growth \%pa $1960 \quad 1970 \quad 1976 \quad 1960-70 \quad 1970-76$

$\begin{array}{llrrrrr} & \text { World }^{\mathbf{c}} & 3350 & 15230 & 24470 & 16.3 & 8.2 \\ 1 & \text { United States } & 1454.9 & 7440.0 & 9083.8 & 17.7 & 3.4 \\ 2 & \text { Soviet Union } & 563.0 & 1876.7 & 2697.8 & 12.8 & 6.2\end{array}$

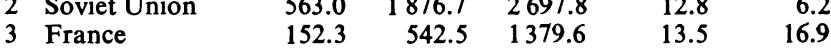

$\begin{array}{llllll}4 \text { Japan } & 24.1 & 436.0 & 1102.9 & 33.6 & 16.7\end{array}$

5 Germany, West $\quad 39.5 \quad 523.3 \quad 1098.5 \quad 29.5 \quad 13.2$

6 United Kingdom $\quad \begin{array}{llllll}180.7 & 604.5 & 914.0 & 12.9 & 7.1\end{array}$

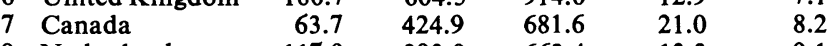

8 Netherlands $\quad \begin{array}{llllll} & 117.0 & 393.0 & 663.4 & 13.3 & 9.1\end{array}$

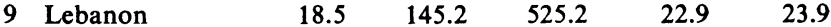

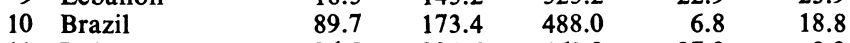

11 Italy

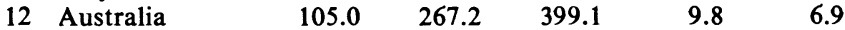

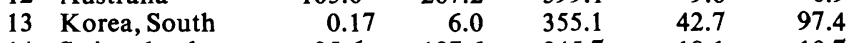

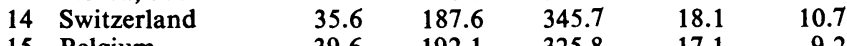

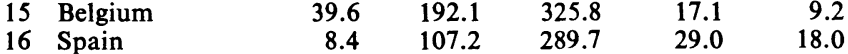

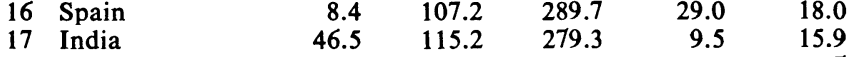

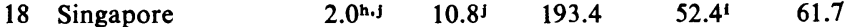

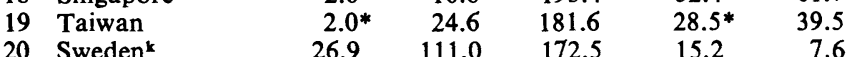

Rest of the worlde

$\begin{array}{llll}\text { Rank } & \text { Tonne-km, net } & \text { Rank } & \text { Tonne-km, net } \\ \text { in } 1976 & & \text { in } 1976 & (\mathrm{mn}) \text { 1976 }\end{array}$

21 South Africa $\quad 160.7 \quad 59=$ Mauritania $^{1} \quad 14.4$

22 Colombia $\quad 154.2 \quad 62=$ Congo $^{1} \quad 14.2$

24 New Zealand $\quad 143.7 \quad 62=$ Poland 14.2

25 Israel $\quad 138.6 \quad 65=$ El Salvador $\quad 14.0$

26 Thailand $\quad 126.8 \quad 65=$ Ivory Coast $^{1} \quad 14.0$

27 Norwayk $\quad 118.9 \quad 67=$ Cent African Emp 13.9

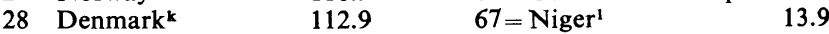

29 Philippines $\quad 111.9 \quad 67=$ Senegal $^{1} \quad 13.9$

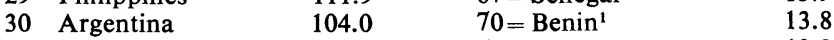

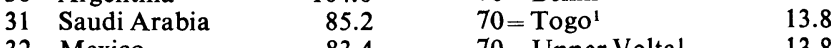

32 Mexico $\quad 83.4 \quad 70=$ Upper Volta $^{1} \quad 13.8$

33 Hongkong $\quad 78.0^{\mathrm{f}} \quad 73$ Costa Rica 13.5

34 Ireland $\quad 77.7 \quad 74$ Afghanistan 13.0

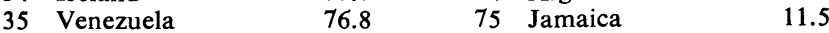

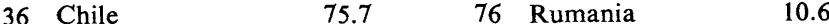

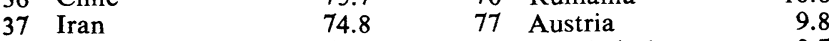

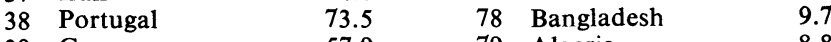

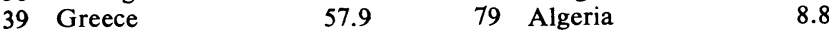

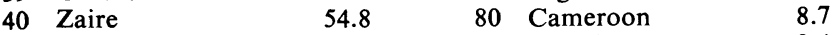

41 Germany, East $\quad \begin{array}{llll}50.5 & 81 & \text { Nigeria } & 8.4 \\ & 47.6 & 82 & \text { Ugandam }\end{array}$

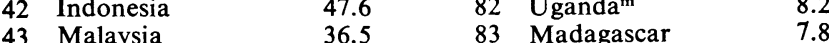

$\begin{array}{lllll}43 & \text { Malaysia } & 36.5 & 83 & \text { Madagascar } \\ 44 & \text { Finland } & 33.1 & 84=\text { Bulgaria } & 7.6\end{array}$

45 Iceland $\quad 30.6 \quad 84=$ Syria $\quad 7.6$

46 Kuwait $\quad 27.1 \quad 86=$ Sudan 7.5

47 Peru $25.9 \quad 86=$ Tunisia

48 Trinidad \& Tobago $24.2 \quad 88$ Ecuador

49 Egypt $\quad 21.9 \quad 89$ Guatemala $\quad 7.1$

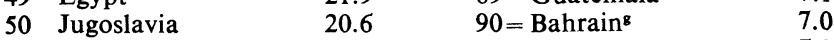

51 Ethiopia $\quad 20.1 \quad 90=$ Oman $^{8} \quad 7.0$

52 Zambia $\quad 19.8 \quad 90=$ Qatar $^{8} \quad 7.0$

53 Kenya $^{\mathrm{m}} \quad 19.7 \quad 90=$ Utd Arab Emirates $^{8} \quad 7.0$

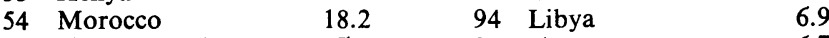

55 Czechoslovakia $\quad 17.4 \quad 95$ Cyprus 6.7

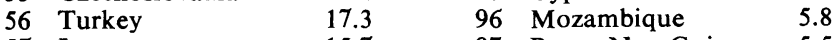

57 Iraq $\quad 15.7 \quad 97$ Papua New Guinea 5.5

58 Chad $^{1} \quad 14.8 \quad 98$ Hungary $\quad 5.2$

$\begin{array}{lrrrr}59=\text { Gabon }^{1} & 14.4 & 99 & \text { Panama } & 5.0 \\ 59=\text { Jordan } & 14.4 & 100 & \text { Malawi } & 4.7\end{array}$

$59=$ Jordan 14.4

-Revenue scheduled traffic (traffic on flights according to a published timetable) of airlines registered in each country. Includes both domestic and international services

'Includes goods, livestock and mail 'Icao members only; excluding China, North Korea and Vietnam ${ }^{\mathrm{d}}$ Countries with over $160 \mathrm{mn}$ passenger-km traffic only ${ }^{\circ} \mathrm{Countries}$ with over $4.5 \mathrm{mn}$ tonne-km traffic only ' 1975 Apportionment of Gulf Air ${ }^{\mathrm{h}} 1966$ '1966-70 'Apportionment of Malaysia-Singapore Airlines Ltd kIncluding apportionment of SAS IIncluding apportionment of Air Afrique (for Gabon until December 2, 1976) mIncluding apportionment of East African Airways Corp (which was disbanded in 1977) 